\title{
Predicting Role of Preoperative Computed Tomography Features on Invasiveness of Thymic Neoplasms
}

\author{
Ulaş Kumbasar ${ }^{1}$, [MD] \\ ORCID: 0000-0003-0616-1326 \\ Onur Taydaş ${ }^{2},[\mathrm{MD}]$ \\ ORCID: 0000-0002-9881-7240 \\ Serkan Uysal ', [MD] \\ ORCID: 0000-0002-1152-2854 \\ Erkan Dikmen ${ }^{1},[\mathrm{MD}]$ \\ ORCID: 0000-0002-0866-5221 \\ Mustafa Yılmaz ${ }^{3},[\mathrm{MD}]$ \\ ORCID: 0000-0002-9881-7240 \\ Metin Demircin ${ }^{1},[\mathrm{MD}]$ \\ ORCID: 0000-0002-4756-3869 \\ Rıza Doğan ',[MD] \\ ORCID: 0000-0003-4845-3044 \\ Macit Arıyürek ${ }^{4},[\mathrm{MD}]$ \\ ORCID: 0000-0002-2966-3321 \\ ${ }^{1}$ Hacettepe University School of Medicine, Department \\ of General Thoracic Surgery \\ 2 Erzincan Binali Yıldırım University Mengücek Gazi \\ Training and Research Hospital, Department of \\ Radiology \\ ${ }^{3}$ Hacettepe University School of Medicine, Department \\ of Cardiovascular Surgery \\ ${ }^{4}$ Hacettepe University School of Medicine, Department \\ of Radiology
}

Corresponding Author: Serkan Uysal

Hacettepe University School of Medicine, Department of General Thoracic Surgery

e-mail: drsuysal@msn.com

Adres: Hacettepe University School of Medicine, Department of General Thoracic Surgery, SıhhiyeAnkara, Turkey

Tel: +905324087481

Received: 14 July 2020, Accepted: 03 August 2020,

Published online: 30 September 2020

\section{wa A B TRAC Ceen}

Objective: The anterior mediastinal masses are composed of neoplasms from many different groups, thymic epithelial tumors being the most common type. Histologic subtype, tumor stage and completeness of resection are considered to be the most important prognostic factors in thymic malignancies. The aim of this study is to evaluate the association between the preoperative Computed Tomography characteristics and the histological types, mostly focusing on thymic neoplasms.

Materials and Methods: Preoperative Computed Tomography features and pathologic data of 40 patients who underwent surgery due to an anterior mediastinal mass were retrospectively analyzed. Patients are grouped as thymomas, thymic carcinomas and others.

Results: Computed Tomography characteristics such as shape, contour, necrosis, lymph node enlargement, surrounding organization, pathologic invasion pattern, internal density, presence of cystic component, pleural/pericardial effusion and calcification were compared with histologic subtypes. Among these parameters solely pathologic invasion and surrounding organization were statistically different between the groups ( $\mathrm{p}<0.001$ and $\mathrm{p}=0.001$, respectively). Correlation between the Computed Tomography and pathologic tumor sizes among histologic subtypes was also analyzed and no statistically significant difference between Computed Tomography and pathologic tumor sizes was detected $(p>0,05)$.

Conclusion: According to the results of our study, Computed Tomography imaging has a limited role in predicting stage and malignant potential particularly for thymic neoplasms and anterior mediastinal masses.

Key words: Thymic neoplasms, computed tomography, preoperative, surgery

\section{INTRODUCTION}

The anterior mediastinal masses are composed of neoplasms from many different groups and constitute about $50 \%$ of all mediastinal masses [1]. The most common anterior mediastinal mass is thymic epithelial tumors (TET), which constitute approximately $20 \%$ of all mediastinal tumors and approximately $47 \%$ of anterior mediastinal masses [2].

Histologic subtype, tumor stage and completeness of resection are considered to be the most important prognostic factors in thymic malignancies. Although most of the patients with early stage 
disease can be cured with complete resection, patients with advanced disease may necessitate neoadjuvant chemotherapy to facilitate complete resection and prolong survival [3-5]. Therefore, the ability to detect patients' stage by radiographic predictors is an important consideration to avoid unnecessary or futile surgeries and help select appropriate patients for adjuvant chemotherapy.

Computed tomography (CT) is the most commonly used imaging method for preoperative evaluation of patients with anterior mediastinal masses which provides important information about tumor size, shape, content, and its relationship to other mediastinal components or borders [6-7]. The purpose of this study is to evaluate the association between the preoperative CT characteristics and the stage, histological types and malignancy, mostly focusing on thymic neoplasms.

\section{MATERIALS AND METHOD}

\section{Patient Selection}

This retrospective study has been approved by the local ethics committee and conducted in accordance with the Declaration of Helsinki (2000). Informed consent was waived because of the retrospective nature of the study. A total of 40 patients who underwent surgery due to an anterior mediastinal mass between October 2007 and December 2016 were included in the study. Patients whose surgical treatment, radiologic imaging or pathologic examination were not available at our hospital were excluded from the study. Patients are grouped as thymomas, thymic carcinomas and others. Others group included anterior mediastinal masses other than thymic pathologies [Granulomatous inflammation $(n=1)$, neuroendocrine tumor $(n=1)$, pericardial cyst $(n=1)$ and vascular malformation $(n=1)]$. CT images and medical records of the patients were retrospectively reviewed.

\section{CT technique}

Patients underwent thorax CT imaging with a 16-detector CT (Somatom Sensation 16, Siemens Medical Systems, Germany) scanner (tube voltage $=130 \mathrm{kV}$, effective $m A s=90$, slice thickness $5 \mathrm{~mm}$, collimation=2x4 mm, pitch=1.6). One hundred milliliters (ml) of intravenous (IV) contrast material $(300 \mathrm{mg} /$ ml Omnipaque, GE Healthcare, Ireland) at a flow rate of $4 \mathrm{ml} / \mathrm{sec}$ was used for CT examinations. The body region between the level of the lung apex and upper pole of kidneys was included in the CT scan area. CT scans were directly sent to the workstation (Centricity Universal Viewer, GE Healthcare, USA).

\section{Image analysis and description of CT findings}

CT images were reviewed by two radiologist experienced in thoracic radiology and were blinded to the surgical, clinical and pathology findings, and radiology reports. Imaging characteristics were determined as size in the largest dimension, shape (oval, round or irregular), contour (smooth, lobulated or irregular), internal density, homogenity, calcification, necrosis or cystic appearance, invasion, pleural or pericardial effusion, capsule integrity, lymphadenopathy, central density and ratio of anteroposterior length to sternum-vertebra distance. The morphological features which were ambiguously determined by the reviewers were reassessed together in a second session, and the exact finding was determined by a consensus decision.

\section{Cinical and Pathological Assessment}

Clinical symptoms, presence of comorbidities and the duration of hospital stay were recorded for all patients. The tumor subtype, pathological size in the largest dimension, surgical margin positivity and microscopic invasion were also recorded as pathological findings.

\section{Statistical Analysis}

Statistical analysis was performed using the SPSS 20.0 for Windows (SPSS, Inc., Chicago, USA) packet program. As a statistical analysis, the categorical variables in the descriptive findings are given in terms of number, percent, and continuous variables are expressed as mean \pm standard deviation and median (smallest, largest value). Pearson Chisquare was used to compare categorical variables. The normal distribution of the data was analyzed by 
Kolmogorov-Smirnov test and Kruskal Wallis, oneway ANOVA and Wilcoxon signed rank tests. The homogeneity of the variances in Anova test was evaluated by Levene Test. In cases where there was a significant difference between the groups, twotailed post-hoc comparisons were made using the Tukey test. Correlation between tumor subtypes and pathologic dimension and correlation between pre and post-operative hemoglobin values was assessed by Pearson or Spearman Correlation tests according to normal distribution fit. The statistical significance level was accepted as $p<0.05$.

\section{RESULTS}

This study consisted of 24 men (64.8\%) and 13 women (35.2\%) with a median age of 51 years (range, 21 - 92 years). Histological subtypes of patients are shown in Table 1. The relationship of the tumor to surrounding tissues was investigated. Eighteen patients $(45 \%)$ had surrounding tissue invasion (Table 2). CT characteristics such as shape (circle, oval, irregular), contour (lobulated, irregular, smooth), necrosis, lymph node enlargement, surrounding organization, pathologic invasion pattern, internal density (homogeneous, heterogeneous), presence of cystic component, pleural/pericardial effusion and calcification were compared with histologic subtypes (Table 3). Among these parameters solely pathologic invasion $(73.3 \%, 85.7 \%$ and 5.7 in thymoma, thymic carcinoma patients and other group, respectively; $\mathrm{p}<0.001)$ and surrounding organization (66.7\%, 85.7\% and 16.7 in thymoma, thymic carcinoma patients and other group, respectively; $p=0.001$ ) were statistically different between the groups.

Table 1. Histological subtypes of patients

\begin{tabular}{|l|c|c|}
\hline Diagnosis & Number & Number \\
\hline Thymic carcinoma & 7 & 7 \\
Thymic cyst & 8 & 8 \\
Thymic hyperplasia & 2 & 2 \\
Follicular hyperplasia & 3 & 3 \\
Thymoma type A & 3 & 3 \\
Thymoma type AB & 2 & 2 \\
Thymoma type B1 & 2 & 2 \\
Thymoma type B2 & 7 & 7 \\
Thymoma type B3 & 1 & 1 \\
Undifferentiated pleomorfic sarkoma & 1 & 1 \\
Granulmatous inflammation & 1 & 1 \\
Neuroendocrine tumor & 1 & 1 \\
Pericardial cyst & 1 & 1 \\
Vascular malformation & & 1 \\
\hline Total & & 1 \\
\hline
\end{tabular}


Acta Medica 2020; 51(3): 18 - 24

Uysal et al.

Table 2. Tissue invasion characteristics of the tumors

\begin{tabular}{|l|c|c|}
\hline Surrounding tissue invasion & $\mathrm{N}$ & $\%$ \\
\hline Mediastinal fat tissue & 7 & 17,5 \\
Capsula & 3 & 7,5 \\
Capsula, Mediastinal fat tissue & 2 & 5,0 \\
Lung, Capsula, Mediastinal fat tissue, lymph node & 1 & 2,5 \\
Lung & 1 & 2,5 \\
Phrenic nerve, Mediastinal fat tissue & 1 & 2,5 \\
Capsula, Pleural metastasis & 1 & 2,5 \\
Mediastinal fat tissue, Lung & 1 & 2,5 \\
Pericard & 1 & 2,5 \\
No invasion & 22 \\
\hline
\end{tabular}

Table3. CT features of patients

\begin{tabular}{|c|c|c|c|c|c|c|c|}
\hline \multirow[t]{2}{*}{ Characteristics } & \multicolumn{2}{|c|}{ Thymoma } & \multicolumn{2}{|c|}{ Thymic carcinoma } & \multicolumn{3}{|c|}{ Others } \\
\hline & $\mathrm{N}$ & $\%$ & $\mathrm{~N}$ & $\%$ & $\mathrm{~N}$ & $\%$ & $p^{*}$ \\
\hline \multicolumn{8}{|l|}{ Shape } \\
\hline Circle $(n=6)$ & 1 & 6,7 & 1 & 14,3 & 4 & 22,2 & \multirow{3}{*}{0,527} \\
\hline Oval $(n=24)$ & 10 & 66,7 & 3 & 42,9 & 11 & 61,1 & \\
\hline Irregular $(n=10)$ & 4 & 26,7 & 3 & 42,9 & 3 & 16,7 & \\
\hline \multicolumn{8}{|l|}{ Contour } \\
\hline Lobulated ( $n=9$ ) & 5 & 33,3 & 2 & 28,6 & 2 & 11,1 & \\
\hline Irregular $(n=15)$ & 6 & 40,0 & 5 & 71,4 & 4 & 22,2 & \\
\hline Smooth $(n=16)$ & 4 & 26,7 & 0 & 0,0 & 12 & 66,7 & \\
\hline \multicolumn{8}{|l|}{ Necrosis } \\
\hline No $(n=29)$ & 12 & 80,0 & 5 & 71,4 & 12 & 66,7 & \\
\hline Necrotic $(n=6)$ & 3 & 20,0 & 2 & 28,6 & 1 & 5,6 & \\
\hline Cystic $(n=5)$ & 0 & 0,0 & 0 & 0,0 & 5 & 27,8 & \\
\hline \multicolumn{8}{|c|}{ Lymph node enlargement } \\
\hline No $(n=35)$ & 13 & 86,7 & 5 & 71,4 & 17 & 94,4 & \multirow{2}{*}{0,293} \\
\hline Yes $(n=5)$ & 2 & 13,3 & 2 & 28,6 & 1 & 5,6 & \\
\hline \multicolumn{8}{|c|}{ Surrounding organization } \\
\hline No $(n=21)$ & 5 & 33,3 & 1 & 14,3 & 15 & 83,3 & \multirow{2}{*}{0,001} \\
\hline Yes $(n=19)$ & 10 & 66,7 & 6 & 85,7 & 3 & 16,7 & \\
\hline \multicolumn{8}{|c|}{ Pleural-Pericardial effusion } \\
\hline No $(n=36)$ & 14 & 93,3 & 6 & 85,7 & 16 & 88,9 & \multirow{2}{*}{0,838} \\
\hline Yes $(n=4)$ & 1 & 6,7 & 1 & 14,3 & 2 & 11,1 & \\
\hline \multicolumn{8}{|l|}{ Pathologic invasion } \\
\hline No $(n=22)$ & 4 & 26,7 & 1 & 14,3 & 17 & 94,4 & \multirow{2}{*}{$<0,001$} \\
\hline Yes $(n=18)$ & 11 & 73,3 & 6 & 85,7 & 1 & 5,6 & \\
\hline \multicolumn{8}{|l|}{ Internal density } \\
\hline Heterogeneous ( $n=15$ ) & 5 & 33,3 & 5 & 71,4 & 5 & 27,8 & 0,118 \\
\hline Homogeneous ( $n=25)$ & 10 & 66,7 & 2 & 28,6 & 13 & 72,2 & \\
\hline \multicolumn{8}{|l|}{ Calcification } \\
\hline No $(n=35)$ & 12 & 80,0 & 6 & 85,7 & 17 & 94,4 & \multirow{2}{*}{0,453} \\
\hline Yes $(n=5)$ & 3 & 20,0 & 1 & 14,3 & 1 & 5,6 & \\
\hline
\end{tabular}

* P values were calculated using the Pearson chi-square test 
Correlation between the CT and pathologic tumor sizes among histologic subtypes are summarized in Table 4. There was no statistically significant difference between $C T$ and pathologic tumor sizes among the histologic groups $(p>0,05)$. Correlation analysis also showed a strong correlation between pathologic and CT tumor sizes (correlation coefficient (r): 0.952). The distribution of pathologic and CT sizes among histologic subtypes are summarized in Table 5.

Table 4. Comparison of CT and pathologic tumor sizes among histologic subtypes

\begin{tabular}{|c|c|c|c|c|c|c|}
\hline & Mean & SD & Median & Min & Max & $p$ \\
\hline \multicolumn{7}{|l|}{ CT size } \\
\hline Thymoma $(n=15)$ & 6,07 & 2,63 & 7,00 & 2 & 10 & \multirow{3}{*}{$0,143^{*}$} \\
\hline Thymic Ca (n=7) & 7,14 & 3,02 & 7,00 & 4 & 11 & \\
\hline Others $(n=18)$ & 5,22 & 3,45 & 4,00 & 2 & 15 & \\
\hline \multicolumn{7}{|l|}{ Pathologic size } \\
\hline Thymoma $(n=15)$ & 6,80 & 2,78 & 7,00 & 2 & 11 & \multirow{3}{*}{$0,154^{*}$} \\
\hline Thymic Ca (n=7) & 9,29 & 4,38 & 8,00 & 4 & 15 & \\
\hline Others $(n=18)$ & 6,13 & 4,24 & 5,00 & 2 & 18 & \\
\hline
\end{tabular}

* Data analyzed by Kruskal Wallis test. CT: Computed Tomography; SD: Standard Deviation; Ca: Carcinoma

Table 5. Correlation analysis of CT and pathologic sizes within histologic subtypes

\begin{tabular}{|c|c|c|}
\hline Histologic subtype & & $\begin{array}{c}\text { Pathologic } \\
\text { size }\end{array}$ \\
\hline \multirow[t]{2}{*}{ Thymoma CT Size } & $r$ & 0,950 \\
\hline & $p$ & $<0,001$ \\
\hline \multirow{2}{*}{ Thymic carcinoma CT size } & $r$ & 0,946 \\
\hline & $\mathrm{p}$ & 0,001 \\
\hline \multirow{2}{*}{ Others CT size } & $r$ & 0,960 \\
\hline & $p$ & $<0,001$ \\
\hline
\end{tabular}

CT: Computed Tomography; r: Correlation Coefficient

\section{DISQUSSION}

The objective of this study was to evaluate which CT characteristics were associated with the pathologic features and malignant potential of anterior mediastinal masses focusing on thymic malignancies. Although we found that some CT parameters were significantly associated with the malignancy of the tumor, most of the features were found to be independent from the malignant potential of thymic tumors. Currently, CT imaging is considered the preferred method for the initial assessment and follow-up for patients with anterior mediastinal masses [8]. In the present study, we primarily focused on the CT features of thymic tumors due to the very limited number of 22 non-thymic anterior mediastinal masses. There are limited studies comparing CT appearance of thymic malignancies with staging of the neoplasm and the results are controversial [9-12]. Although several studies have indicated that CT imaging is not adequate to differentiate the histological subtypes of thymoma, some studies showed that $\mathrm{CT}$ scanning can sufficiently predict histologic classification $[6,8,13,14]$. Some studies indicated that heterogeneous density of the tumor in CT scan was suggestive of higher stage [10,12]. In Shen's study, there was no correlation between the internal density of the tumor and staging [8]. Results of our 
study is similar to Shen's study.

According to Tomiyama's and Priola's studies, occurrence of calcification is higher in stage II/III/IV thymomas than in stage I thymoma [10,12]. However, Harris et al. and Shen et al. found no difference among stages in terms of calcification frequency $[8,15]$. Our results are in consistency with the latter studies.

Ability of CT scan to predict stage status according to invasion into surrounding structures is also debatable. Tomiyama et al. suggested that $\mathrm{CT}$ is a poor predictor of invasion into surrounding tissues [12]. Similarly, Priola et al. highlighted the difficulty of distinguishing between simple adhesion and invasion solely based on CT features [10]. On the contrary, in Marom's study suspicion of infiltration into mediastinal structures is found to be associated with higher stages [9]. Likewise, several studies have shown that the infiltration of surrounding fat suggests an invasive thymoma $[5,16,17]$. Our study has confirmed these studies, which showed a statistically significant association between invasion to nearby structures and malignancy $(p=0,001)$.

In Zhao's study incidence of cyst formation or necrosis is $45.6 \%$ in thymoma patients [5]. In our study occurrence of necrosis is lower with an incidence of $20 \%$ and $28.6 \%$ in thymomas and thymic carcinomas, respectively. We couldn't detect any cystic component in any of our thymic neoplasm patients. According to many studies in the literature, lobulated or irregular tumor contour of the tumor is a predictive of invasiveness $[9,10,12,17]$. In the current study, we were unable to find any association with contour features and tumor invasion or stage. Tumor shape and mediastinal lymph node enlargement is found to be associated with the World Health Organization (WHO) histological classification of thymic malignancies in many studies $[5,8]$.
However, these features are not confirmed as predictive of invasiveness of tumor stage as was also observed in our study $[5,18,19]$. Pericardial or pleural effusion is another CT feature of thymic malignancies which is correlated with staging. In this study, there were no correlation between occurrence of effusion and invasiveness of the tumor.

Although this study was performed in a homogenous cohort of a single institution undergoing resection for anterior mediastinal masses, sample size was relatively small compared to the multicentre studies. This small sample size probably limits the power of the analysis to detect significant associations. Besides, surgically resected patients were included, therefore patients who were already refereed to be unresectable may have been excluded. In addition, this is a retrospective study which may entail inherit problems of selection bias. Prospective studies are needed to verify our results.

In sum, many of the radiological features of thymomas that have previously been investigated and debated such as shape, contour, necrosis, lymph node enlargement, internal density, presence of cystic component, pleural/pericardial effusion and calcification, were found to be not associated with invasiveness and stage of the tumor in our study. Solely, invasion to nearby structures and surrounding organization were significantly correlated with tumor invasiveness and malignancy. Thus, according to our study, CT imaging has a limited role in predicting stage and malignant potential particularly for thymic neoplasms and anterior mediastinal masses.

\section{CONFUCT of INTEREST}

The authors declared no conflicts of interest. 
[1] Strollo DC, Rosado de Christenson ML, Jett JR. Primary mediastinal tumors. Part 1: tumors of the anterior mediastinum. Chest 1997; 112(2):511-22.

[2] Engels EA. Epidemiology of thymoma and associated malignancies. J Thorac Oncol 2010; 5(4):260-5.

[3] Kim ES, Putnam JB, Komaki R et al. Phase II study of a multidisciplinary approach with induction chemotherapy, followed by surgical resection, radiation therapy, and consolidation chemotherapy for unresectable malignant thymomas: final report. Lung Cancer 2004; 44(3):369-79.

[4] Lucchi M, Ambrogi MC, Duranti L et al. Advanced stage thymomas and thymic carcinomas: results of multimodality treatments. Ann Thorac Surg 2005; 79(6):1840-4.

[5] Zhao Y, Chen H, Shi J et al.. The correlation of morphological features of chest computed tomographic scans with clinical characteristics of thymoma. European journal of cardio-thoracic surgery : official journal of the European Association for Cardio-thoracic Surgery 2015; 48(5):698-704.

[6] Tomiyama N, Johkoh T, Mihara N et al. Using the World Health Organization Classification of thymic epithelial neoplasms to describe CT findings. AJR Am J Roentgenol 2002; 179(4):881-6.

[7] Shahrzad M, Le TS, Silva $M$ et al.. Anterior mediastinal masses. AJR Am J Roentgenol 2014; 203(2):128-38.

[8] Shen Y, Gu Z, Ye J et al.. CT staging and preoperative assessment of resectability for thymic epithelial tumors. J Thorac Dis 2016; 8(4):646-55.

[9] Marom EM, Milito MA, Moran CA et al. Computed tomography findings predicting invasiveness of thymoma. J Thorac Oncol 2011; 6(7):1274-81.
[10] Priola AM, Priola SM, Di Franco M et al. Computed tomography and thymoma: distinctive findings in invasive and noninvasive thymoma and predictive features of recurrence. Radiol Med 2010; 115(1):1-21.

[11] Qu YJ, Liu GB, Shi HS et al. Preoperative CT findings of thymoma are correlated with postoperative Masaoka clinical stage. Acad Radiol 2013; 20(1):66-72.

[12] Tomiyama N, Muller NL, Ellis SJ et al. Invasive and noninvasive thymoma: distinctive $\mathrm{CT}$ features. J Comput Assist Tomogr 2001; 25(3):388-93.

[13] Jeong YJ, Lee KS, Kim J et al. Does CT of thymic epithelial tumors enable us to differentiate histologic subtypes and predict prognosis? AJR Am J Roentgenol 2004; 183(2):283-9.

[14] Sadohara J, Fujimoto K, Muller NL et al. Thymic epithelial tumors: comparison of CT and MR imaging findings of low-risk thymomas, high-risk thymomas, and thymic carcinomas. Eur J Radiol 2006; 60(1):70-9.

[15] Harris K, Elsayegh D, Azab at al. Thymoma calcification: is it clinically meaningful? World J Surg Oncol 2011; 9:95.

[16] Chen JL, Weisbrod GL, Herman SJ. Computed tomography and pathologic correlations of thymic lesions. J Thorac Imaging 1988; 3(1):61-5.

[17] Marom EM. Imaging thymoma. J Thorac Oncol 2010; 5(4):296-303.

[18] Hayes SA, Huang J, Plodkowski AJ et al. Preoperative computed tomography findings predict surgical resectability of thymoma. J Thorac Oncol 2014; 9(7):1023-30.

[19] Hu YC, Wu L, Yan LFet al. Predicting subtypes of thymic epithelial tumors using CT: new perspective based on a comprehensive analysis of 216 patients. Sci Rep 2014; 4:6984. 